\title{
Erratum to: Assessing group-level participation in fluid teams: Testing a new metric
}

\author{
Susannah B. F. Paletz • Christian D. Schunn
}

Published online: 18 December 2012

(C) Psychonomic Society, Inc. 2012

\section{Erratum to: Behav Res, Published Vol 43 No 2 June 2011 pp 522 to 536 \\ DOI 10.3758/s13428-011-0070-3}

All the original negative signs in Tables $1,4,5$, and 6 are missing in the published version. The negative signs for Table 1 are necessary for correctly calculating the formula; the negative signs on Tables 4,5 , and 6 are necessary for understanding which relationships are negative versus positive. These errors are on pages 526 (Table 1), 532 (Tables 4 and 5), and 533 (Table 6). The original relevant tables are reproduced below.
Table 1 Steps one through three, utterance-level assignments: one example

\begin{tabular}{|c|c|c|c|c|c|c|c|}
\hline \multirow[t]{2}{*}{ Utterance } & \multirow[t]{2}{*}{ Speaker } & \multicolumn{6}{|c|}{ Person } \\
\hline & & 1 & 2 & 3 & 4 & 5 & 6 \\
\hline Have you received the file yet? & 1 & $1 / 2$ & $-1 / 2$ & 0 & 0 & 0 & 0 \\
\hline No & 2 & $-1 / 2$ & $1 / 2$ & 0 & 0 & 0 & 0 \\
\hline Wait, which file? & 2 & $-1 / 3$ & $2 / 3$ & $-1 / 3$ & 0 & 0 & 0 \\
\hline Have you guys seen the file? & 3 & $-1 / 3$ & $-1 / 3$ & $2 / 3$ & 0 & 0 & 0 \\
\hline No & 2 & $-1 / 3$ & $2 / 3$ & $-1 / 3$ & 0 & 0 & 0 \\
\hline Yes & 1 & $2 / 3$ & $-1 / 3$ & $-1 / 3$ & 0 & 0 & 0 \\
\hline I just sent this great document, & 4 & $-1 / 6$ & $-1 / 6$ & $-1 / 6$ & $5 / 6$ & $-1 / 6$ & $-1 / 6$ \\
\hline Did you guys get it? & 4 & $-1 / 6$ & $-1 / 6$ & $-1 / 6$ & $5 / 6$ & $-1 / 6$ & $-1 / 6$ \\
\hline It's about the new picture we just got. & 4 & $-1 / 6$ & $-1 / 6$ & $-1 / 6$ & $5 / 6$ & $-1 / 6$ & $-1 / 6$ \\
\hline Step 2: Sum across utterances & & -0.83 & 0.17 & -0.83 & 2.50 & -0.50 & -0.50 \\
\hline Step 3: Absolute Value of Step 2 & & 0.83 & 0.17 & 0.83 & 2.50 & 0.50 & 0.50 \\
\hline
\end{tabular}

The online version of the original article can be found at http://dx.doi.org/ 10.3758/s13428-011-0070-3.

S. B. F. Paletz $(\square) \cdot$ C. D. Schunn

University of Pittsburgh,

LRDC 816, 3939 O'Hara Street,

Pittsburgh, PA 15260, USA

e-mail: sbfpaletz@gmail.com 
Table 4 Fixed effects model of fluid/stable team, team composition, and covariates on $P_{s}$

${ }^{+} .10<p<.05, * p<.05$, $* * p<.01, * * * p<.001$

Table 5 Fixed effects model of fluid/stable team, team composition, and covariates on coded participation

${ }^{+} .10<p<.05, * p<.05$,

$* * p<.01, * * * p<.001$

Table 6 Fixed effects model of fluid/stable team, team composition, and covariates on standard deviation of participation

${ }^{+} .10<p<.05, * p<.05$, $* * p<.01, * * * p<.001$

\begin{tabular}{lccccc}
\hline Independent Variable & $\gamma$ Coefficient & $S E$ & $t$ & $d f$ & $p$ \\
\hline Covariates & & & & & \\
Number of utterances per block & -0.007 & 0.002 & $-4.45^{* * *}$ & 531 & $<.001$ \\
Mission science team (A vs. B) & 0.007 & 0.031 & 0.25 & 110 & .81 \\
Early/later in first 90 days & 0.054 & 0.029 & $1.85^{+}$ & 110 & .067 \\
Predictors & & & & & \\
Fluid vs. stable teams & -0.027 & 0.019 & -1.43 & 531 & .15 \\
All males versus mixed-gender & -0.002 & 0.025 & -0.08 & 531 & .94 \\
Homogenous older versus mixed-age groups & -0.042 & 0.048 & -0.88 & 531 & .38 \\
Homogeneous younger versus mixed-age groups & -0.084 & 0.031 & $-2.70^{* *}$ & 531 & .008 \\
Presence of negative affect & 0.040 & 0.017 & $2.39^{*}$ & 531 & .017 \\
\hline
\end{tabular}

\begin{tabular}{llcccc}
\hline Independent Variable & $\gamma$ Coefficient & $S E$ & $t$ & & $d f$ \\
\hline Covariates & & & & & \\
Number of utterances per block & -0.415 & 0.152 & $-2.73^{* *}$ & 531 & .007 \\
Mission science team (A vs. B) & -1.478 & 2.876 & -0.51 & 110 & .61 \\
Early/later in first 90 days & 1.859 & 2.896 & 0.64 & 110 & .52 \\
Predictors & & & & & \\
Fluid vs. stable teams & -1.470 & 1.797 & -0.82 & 531 & .41 \\
All males versus mixed-gender & -1.098 & 2.328 & -0.47 & 531 & .64 \\
Homogenous older versus mixed-age groups & -1.352 & 4.866 & -0.28 & 531 & .78 \\
Homogeneous younger versus mixed-age groups & -5.150 & 2.654 & $-1.94^{+}$ & 531 & .052 \\
Presence of negative affect & 4.934 & 2.044 & $2.41^{*}$ & 531 & .016 \\
\hline
\end{tabular}

\begin{tabular}{lccccc}
\hline Independent Variable & $\gamma$ Coefficient & $S E$ & $t$ & $d f$ & $p$ \\
\hline Covariates & & & & & \\
Number of utterances per block & -0.005 & $<0.001$ & $-4.93^{* * *}$ & 531 & $<.001$ \\
Mission science team (A vs. B) & -0.030 & 0.023 & -1.33 & 110 & .19 \\
Early/later in first 90 days & 0.041 & 0.188 & $2.17^{*}$ & 110 & .032 \\
Predictors & & & & & \\
Fluid vs. stable teams & -0.028 & 0.010 & $-2.75^{* *}$ & 531 & .007 \\
All males versus mixed-gender & 0.028 & 0.017 & $1.71^{+}$ & 531 & .088 \\
Homogenous older versus mixed-age groups & 0.035 & 0.026 & 1.32 & 531 & .19 \\
Homogeneous younger versus mixed-age groups & -0.010 & 0.021 & -0.49 & 531 & .63 \\
Presence of negative affect & 0.027 & 0.011 & $2.54^{*}$ & 531 & .012 \\
\hline
\end{tabular}

